\title{
Endoscopic-Assisted Transvesical Laparoscopy: A New Paradigm in Minimally Invasive Bladder Surgery
}

\author{
Yu Guang Tan, MBBS, MRCS \\ Department of Urology, Singapore General Hospital, Singapore, Singapore. \\ E-mail: yuguangtan90@gmail.com
}

Tze Kiat Ng, MBBS, MRCS, FAMS

Department of Urology, Singapore General Hospital, Singapore, Singapore.

John S.P. Yuen, MBBS, MMed, MRCS, FAMS, DPhil

Department of Urology, Singapore General Hospital, Singapore, Singapore.

(c) Mary Ann Liebert, Inc. DOI: 10.1089/vid.2019.0100
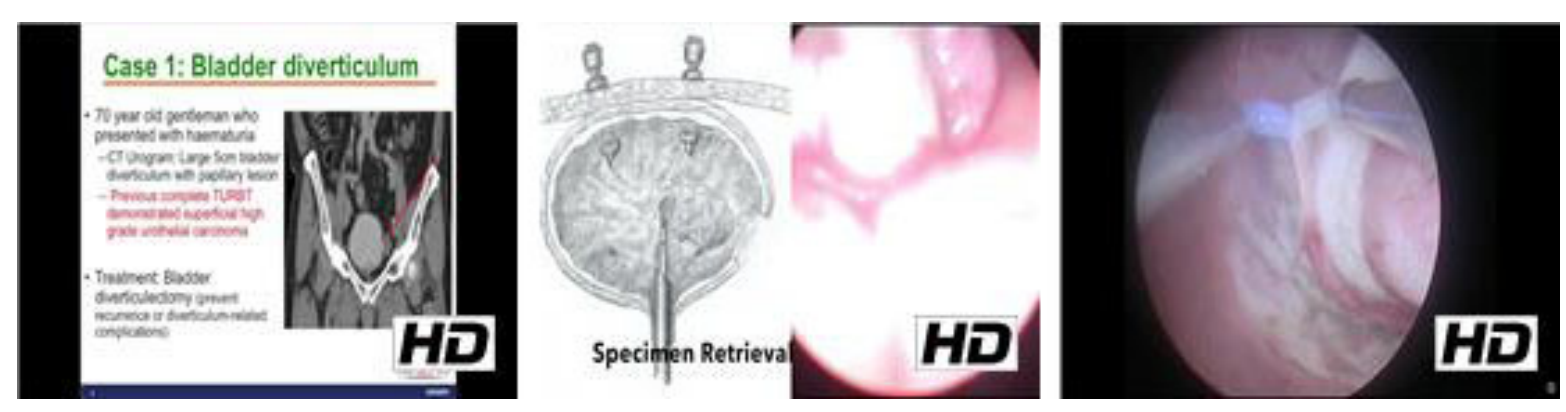

\begin{abstract}
Introduction: Transurethral endoscopic surgery is limited by its incompatibility in defect repair that requires suturing. Open surgery carries significant higher morbidities, whereas pneumoperitoneum in conventional laparoscopy precludes patients with significant cardiovascular and respiratory diseases. There had been technical reports of transvesical laparoscopy in bladder diverticulectomy, employing the conventional three-port triangulation technique; however, this technique results in multiple sizeable bladder defects that predisposes to prolonged urinary extravasation. The objective of this report is to present the first endoscope-assisted transvesical laparoscopy (EtLap) technique with just two small suprapubic 5-mm port in performing resection of various benign bladder pathologies.
\end{abstract}

Materials and Methods: We present a single-surgeon series of EtLap excision of three benign bladder conditions: diverticulum, urachal nodule, and trigonal mass. The materials for the video and illustration were primarily based on the bladder diverticulectomy. A step-by-step EtLap diverticulectomy technique is as follows: (1) patient is placed in lithotomy position, (2) cystoscopy is performed to observe the diverticular opening in relation to the ureteral orifices, (3) two suprapubic 5-mm self-retentive laparoscopic balloon ports were inserted under transabdominal ultrasound guidance and cystoscopic vision into the bladder, (3) creation of pneumovesicum (12 mm Hg) is achieved by draining of irrigation fluid through the resectoscope, whereas $\mathrm{CO}_{2}$ is infused into the bladder through one of the 5-mm working ports, (5) the bladder pathology is resected by a combination of transurethral resectoscopic and transvesical laparoscopic dissections using instruments such as hook and spade, (6) the transurethral natural orifice access provides for illumination, visualization, dissection, specimen retrieval, and sutures passage, (7) bladder defect repair is by laparoscopic continuous suturing technique with a 3-O barbed suture (RB-1 needle), and (8) closure of the small 5-mm port bladder defects is achieved by looping 3-O Vicryl sutures subcutaneously with a suture passer.

Results: Three patients had undergone the earlier described surgery for (1) diverticulectomy (a 5-cm left lateral wall diverticulum that contained a small pTa papillary transitional cell carcinoma (TCC) 
previously resected endoscopically with no macroscopic disease. The indication for diverticulectomy was to prevent tumor recurrence and diverticulum-related complications); (2) full-thickness wide excision of an urachal nodule at bladder dome; and (3) excision biopsy of a 2-cm trigonal mass. Mean operating time was 150 minutes (range 110-180) with minimal blood loss. Mean hospital stay was 2 days (range 1-3). All patients had urinary catheter removal at postoperative day 7 with prior cystograms showing no urinary leakage. There were no complications reported. All patients had only two small healed aesthetically looking 5-mm port suprapubic wounds. Final histology of the specimens showed the diverticulum containing small residual pTa TCC with clear margins, and benign urachal and trigonal nodules.

Conclusion: This is the first description of an endoscope-assisted transvesical laparoscopic technique for minimally invasive bladder surgery using just two 5-mm suprapubic ports. It fully utilizes the transurethral resectoscope as a natural-orifice access to the surgical site to provide illumination, observation, operative maneuvers (traction, incision, dissection, and suction), suture passage, and specimen retrieval. It represents a safe, novel, and intuitive approach to resection of various benign bladder pathologies.

No competing financial interests exist.

Runtime of video: 6 mins 37 secs

Keywords: transvesical laparoscopy, bladder diverticulectomy, endoscopic-assisted surgery, natural transurethral orifice

\section{Cite this video}

Yu Guang Tan, Tze Kiat Ng, John SP Yuen, Endoscopic-Assisted Transvesical Laparoscopy: A New Paradigm in Minimally Invasive Bladder Surgery, Videourology. 2020, DOI: 10.1089/vid.2019.0100.

Original Publication Date: 2020 